\title{
Exploring the oceanic microeukaryotic interactome with metaomics approaches
}

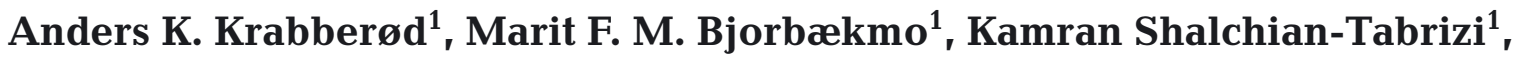 \\ Ramiro Logares ${ }^{2,1, *}$ \\ ${ }^{1}$ University of Oslo, Department of Biosciences, Section for Genetics and Evolutionary Biology (Evogene), Blindernv. 31, \\ 0316 Oslo, Norway \\ ${ }^{2}$ Institute of Marine Sciences (ICM), CSIC, Passeig Marítim de la Barceloneta, Barcelona, Spain
}

\begin{abstract}
Biological communities are systems composed of many interacting parts (species, populations or single cells) that in combination constitute the functional basis of the biosphere. Animal and plant ecologists have advanced substantially our understanding of ecological interactions. In contrast, our knowledge of ecological interaction in microbes is still rudimentary. This represents a major knowledge gap, as microbes are key players in almost all ecosystems, particularly in the oceans. Several studies still pool together widely different marine microbes into broad functional categories (e.g. grazers) and therefore overlook fine-grained species/population-specific interactions. Increasing our understanding of ecological interactions is particularly needed for oceanic microeukaryotes, which include a large diversity of poorly understood symbiotic relationships that range from mutualistic to parasitic. The reason for the current state of affairs is that determining ecological interactions between microbes has proven to be highly challenging. However, recent technological developments in genomics and transcriptomics (metaomics for short), coupled with microfluidics and high-performance computing are making it increasingly feasible to determine ecological interactions at the microscale. Here, we present our views on how this field will advance thanks to the progress in metaomics approaches as well as potential avenues for future research.
\end{abstract}

KEY WORDS: Microeukaryotes · Interactions · Single-cell genomics · Single-cell transcriptomics · High-throughput sequencing

\section{THE MICROBIAL OCEAN}

Our collective awareness of the significance of marine ecosystems has increased steadily during the last $40 \mathrm{yr}$, progressing in concert with the development of new technologies that have allowed us to dig deeper into the microbial world. A number of key events can be identified from this period, for example the formulation of the microbial-loop concept, the discovery of

${ }^{*}$ Corresponding author: ramiro.logares@gmail.com abundant photoautotrophic (Synechococcus and Prochlorococcus) and heterotrophic (SAR11) pico-sized prokaryotic plankton as well as novel lineages of heterotrophic picoeukaryotes (e.g. MALV and MAST), the recognition of the importance of Archaea in oceanic plankton, the realization that the majority of marine microbes cannot be cultured, the discovery of the rare biosphere and the key role of viruses in oceanic communities (Kirchman 2008).

() The authors 2017. Open Access under Creative Commons by Attribution Licence. Use, distribution and reproduction are unrestricted. Authors and original publication must be credited. 
Most of these findings were initiated by a revolution that started $\sim 25$ yr ago with the introduction of molecular methods to marine microbial ecology. This revolution has generated a wealth of new knowledge, and during the last $8 \mathrm{yr}$, it has gained renewed momentum with the advent of highthroughput sequencing platforms (Logares et al. 2012, Goodwin et al. 2016) and a variety of other high-performance approaches such as genomics, transcriptomics, metagenomics, metatranscriptomics, proteomics and single-cell genomics (metanomics for short). High-throughput sequencing has been a key addition to the molecular toolbox of microbial ecologists, allowing a deeper exploration of microbial diversity, distributions and metabolic functions. Questions that were impenetrable a decade ago are now being addressed, such as the magnitude of microbial diversity and its spatiotemporal patterns (Sogin et al. 2006, Galand et al. 2009, Pedrós-Alió 2012, Logares et al. 2013, 2014a, 2015, Rinke et al. 2013, Locey \& Lennon 2016, Schloss et al. 2016). In addition, the introduction of high-performance metaomics techniques and the generation of big data is encouraging microbiologists to embark upon challenging studies which aim to understand the functioning of increasingly complex eco-systems.

\section{MOVING TOWARD OCEANIC ECO-SYSTEMS BIOLOGY}

Despite the overall progress, there is a long road ahead when moving from simplistic to holistic models of oceanic ecosystem functioning. Such a systems biology approach, which aims at understanding ecosystems as a whole by considering all molecular and ecological interactions that ultimately contribute to its functioning and which constitute the interactome, is fundamental for our understanding of the global ocean as a large integrated system that regulate important parameters in the biosphere. To achieve such holistic understanding, at least 2 types of ecosystem data are needed: (1) a list of components, represented by species or populations, and (2) the interactions between these components in a spatiotemporal context (Raes \& Bork 2008). In microbes, the use of metanomics has contributed substantially to increase our understanding of Point 1: species or population diversity within communities. Still, most microbial species remain unknown or poorly known because they are currently unculturable, limiting our access to their genomes, gene- expression patterns or metabolisms using standard techniques. Much less is known regarding Point 2: interactions between microbial species or populations within ecosystems. Here, interactions may refer to classic ecological interactions (e.g. competition) (Box 1), structural interactions (e.g. one microbe providing physical support for another) as well as metabolic interactions among organisms (e.g. metabolic cooperation) (Raes \& Bork 2008). Ecological interactions between microbes can be negative (antagonistic), positive (synergistic) or neutral for the species involved (Lidicker 1979, Leadbetter \& Poindexter 1986, Faust \& Raes 2012) (Fig. 1, Box 1).

Although these definitions are widely used for categorizing organism's life modes, the boundaries between them in real-life can be blurred, as in parasitism vs. mutualism (Box 1) (Stat et al. 2008). Symbiosis (sensu lato) (Box 1) may be very common in marine planktonic protists (López-García \& Moreira 2008), and the character of the relationship between host and symbiont can change not only in the course of evolution but also during the host's lifetime (Wagner-Döbler et al. 2010). Competition is the classic example where both species involved are negatively impacted by the interaction, while mutualism is its counterpart, bringing benefits for both. Parasitism and predation represent typical examples of win-loss interactions, where one of the symbionts gets all the benefits and the other assumes all the costs (Fig. 1, Box 1). Commensalism and amensalism are opposites too, with commensalism bringing benefits to one species at no cost for the other, while in amensalism, one of the interacting species is negatively impacted by another with no benefits to itself (Fig. 1). Microbial interactions may require physical contact between the cells involved (e.g. symbiosis) or not. Within microbial communities, interactions that do not require physical contact can be important for instance when species release secondary metabolites that inhibit the growth of other taxa (negative allelopathy) (Legrand et al. 2003) or when released metabolic by-products turn out to be essential for other members of the community (e.g. vitamins) (Sañudo-Wilhelmy et al. 2014). Although several experiments and field studies have investigated microbial intra-/inter-specific competition (Fredrickson \& Stephanopoulos 1981), predation (Guerrero et al. 1986, Berney et al. 2013), parasitism (Chambouvet et al. 2008), mutualism/symbiosis (Gast \& Caron 1996), and detritivory (Kirchman 2008), the majority of interactions among marine microbes is still unknown. 
Box 1. Ecological interactions from a microbial perspective

Symbiosis in its broadest sense refers to 2 biologically different species living together in a close and long-term relationship (de Bary 1879, Wilkinson 2001, Martin \& Schwab 2013). Different types of symbiotic relationships are commonly categorized into overlapping categories ranging from those that are harmful for one or both of the partners (i.e. competition and parasitism) to those where both partners benefit from the interaction (e.g. mutualism; Fig. 1).

Holobiont refers to a host and all its symbiotic partners (Margulis \& Fester 1991). A classic example of a holobiont would be a coral reef, which consists not only of the host and the dinoflagellate photosymbionts but also of marine fungi and other microorganisms (Amend et al. 2012, del Campo et al. 2016).

Mutualism is a symbiotic relationship in which both partners benefit from the association. The degree to which the symbionts in the mutualistic relationship depend on each other can range from short-term interactions to obligatory symbiosis where one or both partners are unable to survive without the other. In the most extreme cases, one of the partners becomes so dependent on its host that it transfers some of its genetic material to the host nucleus while reducing its own genome, which has happened with the fully assimilated cyanobacteria that has turned into a chloroplast in phototrophic eukaryotes. Another example of mutualistic photosymbiosis in the marine environment is the obligate interaction between corals and their co-evolved dinoflagellate photosymbionts: Symbiodinium spp. (Stat et al. 2008). Photosymbiosis is also a common phenomenon in planktonic protists, such as radiolarians, foraminiferans, ciliates, dinoflagellates, and diatoms (Anderson 1983, Pawlowski et al. 2001, Nowack \& Melkonian 2010, Siano et al. 2010, Gómez et al. 2016, Mordret et al. 2016).

Parasitism is a type of symbiosis in which one of the symbionts benefits at the expense of the other. Parasitic life strategies have evolved independently many times in the evolution of microeukaryotes, and parasites can be found in most of the microeukaryotic taxonomic super groups (Patterson 1999, Skovgaard 2014, Poulin \& Randhawa 2015). Viruses could also be included in the parasite category (Box 2). Our knowledge of parasite diversity is highly biased. Within the eukaryotes, the majority of described parasitic species are encountered in conspicuous, and often medicinally or economically important, animal taxa. While plant parasitic insects represent $64 \%$ of named parasites, parasitic protists, fungi, and plants comprise only $7 \%$ of the named eukaryotic parasitic species (de Meeûs \& Renaud 2002). Recently, Jephcott et al. (2016) showed that when parasites were added to food web analyses, they increased connectance and linkage density (Box 3). Interestingly, Lima-Mendez et al. (2015) reported that parasitic interactions were the most abundant feature in their global-ocean interaction network, with a predominance of marine alveolates (Box 4).

Predation is, like parasitism, an interaction that has a positive effect for one of the partners involved, while the other partner is affected negatively. Predators have probably been present since the first ecosystems developed on Earth, with bacteria killing other bacteria for nutritional purposes. Fossil data and molecular clock estimates have shown that predator microeukaryotes feeding on bacteria were present 1200 million years ago (Mya) (Parfrey et al. 2011, Knoll 2014, Porter 2016). About $800 \mathrm{Mya}$, there was a large diversification and rapid evolution of microeukaryotes comparable to the Cambrian explosion of metazoan diversity. This microeukaryotic diversification is consistent with the appearance of eukaryovory (eukaryotes feeding on other eukaryotes) that evolved independently in several eukaryotic clades in the same time period (Sherr \& Sherr 2002, Porter 2016).

Competition is a classic concept in ecology and evolution (Darwin 1859, Gause 1934). It was invoked to understand the differences in reproductive strategies between species that either produce a lot of offspring, exploiting many different niches, and those that invest heavily in a few offspring maximizing their chance of survival (MacArthur \& Wilson 1967). Studies on microbes in cultures show that when populations of different species occupy the same environment or niche, they compete for the same resources, and this, in turn, will lead to a negative effect on both species involved. Occasionally, competition will lead to the local extinction of one of the populations, especially when it is focused on a single resource and populations have no other form of interaction (Fredrickson \& Stephanopoulos 1981).

\section{UNDERSTANDING OCEANIC MICROBIAL ECO-SYSTEMS AS INTERACTION NETWORKS}

To date, most studies on oceanic microbial interactions have focused on specific relationships, providing limited insight into the complex network of ecological interactions occurring in communities that together sustain ecosystem functioning. The tradi-

Fig. 1. Possible ecological interactions between 2 individuals. The result of the interaction for each member of the pair can be positive, negative or neutral. For example, in predation, one partner obtains the benefits while the other assumes the costs. Based on Faust \& Raes (2012)

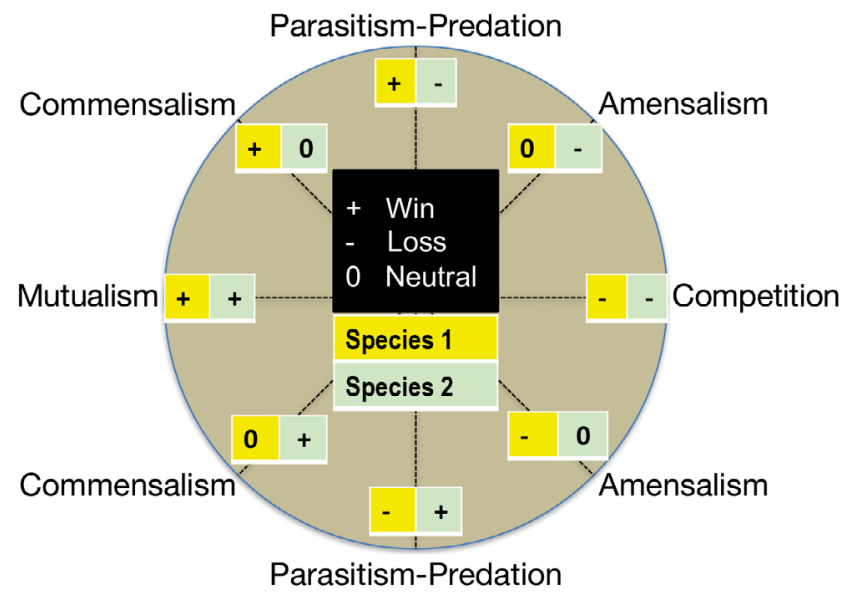


Box 2. Viruses, a special kind of parasitism?

\begin{abstract}
Viral relationships with other microeukaryotes can be regarded as parasitism because their success is dependent on the negative effects on another organism that they infect. Viruses are prevalent in the oceans, reaching abundances of millions per millilitre of seawater (Breitbart 2012). They hold key roles in oceanic ecosystem functioning by limiting microbial population sizes through lysis, reprogramming of host metabolism, and horizontal gene transfer (Rohwer \& Thurber 2009, Brum et al. 2015). Up to $20 \%$ of the marine microbial plankton is exposed to viral lysis each day; therefore, viruses contribute significantly to the turnover of nutrients and carbon in the oceans, where they may induce the release of $\sim 10$ billion tons of carbon daily (Suttle 2007). In globalocean interactome analyses, Lima-Mendez et al. (2015) found a high host specificity in marine viruses, with $43 \%$ of the phages interacting with a single species and the other $57 \%$ interacting with a few species which were normally closely related. Single-cell genomics (Fig. 4) is particularly well-suited for detecting viruses and their protist hosts (Yoon et al. 2011). Recent technical improvements increase the throughput of viral-infection screening of cells (protists or prokaryotes) by hybridizing multiple displacement amplification products generated through typical single-cell genomics procedures (Fig. 4) against multiple viral genomes immobilized on a microarray (Martínez-García et al. 2014).
\end{abstract}

tional approach has been to look primarily at feeding habits, establishing relationships between functional groups (Fig. 2). In a nutshell, the traditional marine microbial food chain is composed of primary producers that generate organic matter through photosynthesis as well as a large number of heterotrophic species that feed, directly or indirectly, on the organic carbon produced by them (DeLong \& Karl 2005). A key alternative carbon and energy pathway is the so-called microbial loop, in which prokaryotes absorb dissolved organic carbon from the environment and reintroduce it into the classic food chain through heterotrophic flagellates (eukaryotes) that prey on them (Fig. 2). Viruses attack and eventually kill several members of this community (Box 2), releasing nutrients, complex molecules, and organic matter that is either recycled in the sunlit community or exported to darker deeper waters. All the players in this food web contribute to the pool of dissolved organic carbon.

The traditional food chain (Fig. 2) has certainly been useful as a starting point for understanding the functioning of marine microbial communities, but it needs to be upgraded to a more detailed description that integrates species, populations, and their specific interactions. Historically, the interactions between symbionts and hosts have been a neglected component of studies addressing food web dynamics. Recent research has shown that inclusion of parasites in food webs increases diversity and complexity and thereby alters the structure of food web topologies (Amundsen et al. 2009, Dunne et al. 2013, Jephcott et al. 2016). The majority of studies that have included parasites in food web analyses have only dealt with macroparasites, while microparasites, such as specific microeukaryotes, have been ignored to a large degree.

During the last decades, ecologists have started to substitute simple linear food-chain models with interactions networks, which represent better the complexity of natural systems. One of the main obstacles for using a network approach on microbial populations has been the technological limitation for identifying (1) all network components (microbial populations or species) and (2) their ecological interactions. Determining network components is not straightforward, as delineating microbial populations or species is normally a complicated task (Logares 2006). The pragmatic solution to this problem is to use operational taxonomic units (OTUs), which may or may not agree with biological species, but which still allow us

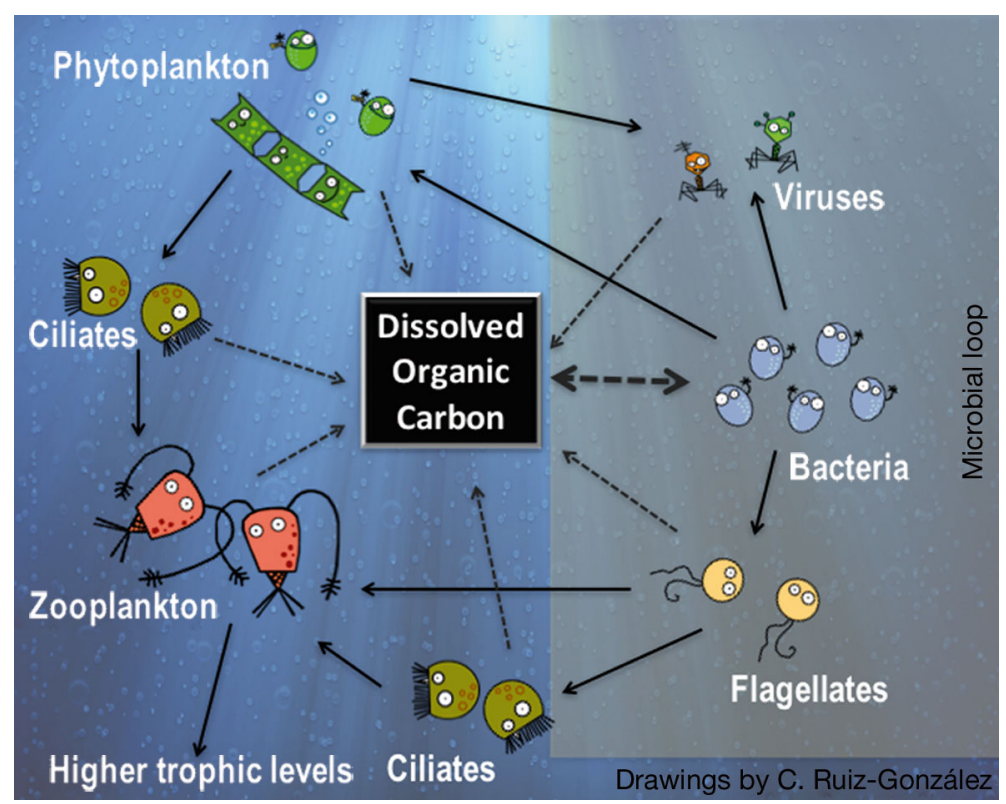

Fig. 2. Simplified microbial food web in the sunlit ocean. Left side: classic description of the carbon flow from photosynthetic algae to grazers and higher trophic levels in the food chain. Right side: microbial loop, with bacteria using dissolved organic carbon to gain biomass, which then re-enters the classic carbon flow through protists. Based on DeLong \& Karl (2005) 
to have units that can be used in downstream analyses (Logares et al. 2012). Based on correlations and anti-correlations of the OTUs, it is possible to build association networks (Box 3), which can be used to generate hypotheses about which organisms may be interacting, both positively or negatively (Faust \& Raes 2012). Yet, one network could have 2000 or 20000 OTUs (nodes) depending on whether a threshold of 97 or $99 \%$ similarity was used for clustering $16 \mathrm{~S}$ or $18 \mathrm{~S}$ rDNA data into OTUs (Logares et al. 2015) or depending on whether different clustering algorithms were used (Edgar 2013, Sinclair et al. 2015). In microeukaryotes, different rates of evolutionary divergence between groups (Pernice et al. 2013) complicate the application of a single threshold of divergence to delineate equivalent ecological units or OTUs. This issue of defining units (as populations or species) permeates many studies in microbial ecology, yet strong patterns across microbial communities are normally detected independently of specific methods or algorithm parameters.

In association networks, OTUs (nodes) are connected through edges, representing ecological interactions or associations between organisms (Fig. 3, Box 3). The topology of the network has properties which may help to understand the role of the species in the ecosystem (Bascompte 2009). For example,

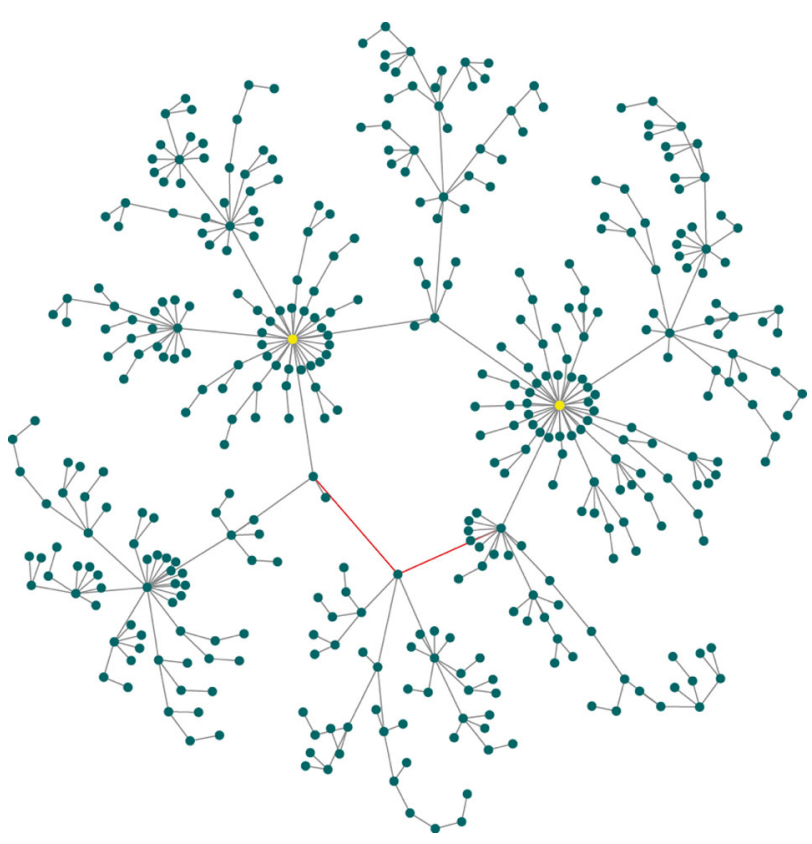

Fig. 3. Hypothetical network of species interactions or associations. Here, species are the nodes, while the links (edges) represent associations or ecological interactions. Nodes with several links (edges) are hubs (i.e. species with many interactions in the network [yellow nodes]). Grey edges represent positive interactions (co-occurrence, e.g. photosymbiosis), while red edges represent negative ones (co-exclusion, e.g. competition)

Box 3. Networks (based on Pascual \& Dunne 2006)

Basic definitions:

Connectivity: a network is 'connected' when it is possible to reach each node from any other node (following edge [i.e. link] directionality if relevant). Otherwise, the network is 'not connected'.

Association network: network built using positive or negative correlations between taxa. Here, edges indicate positive or negative correlations, not necessarily ecological interactions.

Ecological network: representation of real-world ecological relationships. Here, nodes (species) are connected through directional or non-directional edges which represent proved ecological interactions.

Network properties:

Degree of a node: number of edges connected to a node (or number of correlations/interactions connected to a species). Degree distribution: cumulative distribution of node's degrees (i.e. cumulative distribution of the number of links per species).

Hubs: nodes with a high degree in a network.

Distance $\left(\boldsymbol{d}_{i j}\right)$ : shortest distance between nodes $i$ and $j$, i.e. distance that involves connecting nodes $i$ and $j$ through a path that involves crossing the minimum amount of edges.

Characteristic path length $(\boldsymbol{D})$ : mean number of steps (edges) along the shortest paths for all node-pairs in a network, i.e. distance $(d)$ average.

Connectance $(\boldsymbol{C})$ : the proportion of observed edges as compared to all possible edges. $\boldsymbol{L} / \boldsymbol{S}^{2}$, where L: links, S: species. Complexity or linkage density: mean number of edges per node.

Clustering coefficient: measures the degree of clustering of the nodes in a network. It represents the fraction of realized links between neighbors of a node (out of all possible neighbor pairs) averaged over all nodes. In real networks, this quantity tends to be larger than in random networks.

Small-world networks: networks featuring a small $D$ present a 'small-world' behavior. If networks also feature a large clustering coefficient, then they display a 'strong' small-world behavior (Watts \& Strogatz 1998). 
networks may feature nodes with a large number of edges connected to them (i.e. nodes with a high degree; see Box 3). These nodes may represent keystone species that could influence a large number of other species in the same ecosystem. If such highly connected species are lost, the network may break into disconnected sub-webs (Montoya et al. 2006). If the network features a scale-free topology, this implies a highly uneven degree distribution (Box 3), with many nodes having a low degree and very few having a very high degree. The latter pattern of node interconnection is more robust to random removal of species, increasing the robustness of an ecosystem or community against ecological disturbance. Networks that have a small characteristic path length and a high degree (Box 3) are regarded as small-world networks. In these networks, most nodes can be reached from every other node in a few numbers of steps (Watts \& Strogatz 1998, Barabasi \& Albert 1999, Pascual \& Dunne 2006).

During the last $5 \mathrm{yr}$, multiple studies have analyzed microbial networks. In particular, 2 studies reconstructed association networks from a marine-coastal site that suggested novel relationships of unknown type between protists and also indicated that virusbacteria relationships were more specific than protist-bacteria relationships (Steele et al. 2011, Chow et al. 2014). More recently, a large-scale open-ocean study from the TARA oceans expedition (Karsenti et al. 2011) predicted 81590 biotic interactions, of which $72 \%$ were positive and $28 \%$ negative (LimaMendez et al. 2015). Most of the positive correlations were between putative parasitic Syndiniales (marine alveolates, MALVs; Box 4) and either dinoflagellates or tintinnids. However, results regarding putative interactions based on rDNA alone must be interpreted with caution. It has been shown that MALVs are more abundant in the rDNA than in the rRNA pool (Not et al. 2009, Massana et al. 2015), and eukaryotes have several copies of the rDNA operon per genome (Zhu et al. 2005, Decelle et al. 2014). Therefore, the number of interactions could be inflated due to either multiple rDNA copy numbers as well as non-functional or extracellular rDNA. In addition, association networks from Lima-Mendez et al. (2015) predicted multiple positive associations between bacteria and protists. They found associations that have earlier been found in cultures, e.g. between diatoms and Flavobacteria (Jolley \& Jones 1977) and between dinoflagellates and Rhodobacterales (Miller \& Belas 2004), as well as interactions that have previously been inferred by single-cell genomics (Martínez-García et al. 2012).

Even though association networks are powerful tools for generating hypotheses, they fall short in proving the exact nature of the ecological interaction. In order to determine that an ecological interaction is on-going as well as to infer the type of interaction, other methods should be used together with association networks. Such methods could range from microscopy to experimental culturing, yet here we will focus on how metaomics techniques can be coupled with association networks to determine interaction occurrence and type.

Box 4. The case of marine alveolates

One important case of a symbiotic relationship not fully understood, but tentatively taken to be one of parasitism, is that of the marine alveolates (MALVs), an enigmatic group of protists situated between Perkinsea and Dinophycea within Alveolata (Guillou et al. 2008). The MALVs started to appear in early environmental libraries of marine plankton from multiple oceanic locations (Moon-van der Staay et al. 2001, Not et al. 2007, Guillou et al. 2008, López-García \& Moreira 2008, Massana 2011). During the last decade and a half, it has been increasingly apparent that the diversity and distribution of this group is much larger than previously thought. They are among the most diverse groups in the pico-plankton fraction $(<3 \mu \mathrm{m})$ of the global ocean (de Vargas et al. 2015). Little is known about the morphology and ecological role of MALVs. Few species have been described, and these are all parasitic, infecting a wide array of hosts, such as dinoflagellates, copepods, ciliates, crabs and fish (Harada et al. 2007, Guillou et al. 2008, Skovgaard \& Daugbjerg 2008, Skovgaard et al. 2009). Recent work using multiple displacement amplification on isolated single cells of radiolarians has revealed a surprisingly large MALV diversity and several distinct groups (Krabberød et al. 2017). One MALV group was associated only with polycystine radiolarians, another group was associated with a mixture of radiolarian and phaeodarian host species, and an additional group was associated only with a single phaeodarian host (Bråte et al. 2012, Ikenoue et al. 2016). The nature of the relationship between MALVs and radiolarians is not known, but given that all analysed MALV species are heterotrophic, and that there is no known photosynthetic species within the clade, the relationship is most likely to be one of parasitism or commensalism.

Further studies using new as well as established techniques are needed to determine the role of MALVs in ecosystems. In particular, it has to be determined what sort of symbiotic relationship there is between the radiolarian host and MALVs. In many of the studies published so far the MALV symbiont has never been observed, and its presence has only been inferred by molecular methods (Dolven et al. 2007, Bråte et al. 2012, Ikenoue et al. 2016). A study of the ultrastructure of the MALV symbiont as well as its ecological role is therefore much needed. 


\section{USING METAOMICS TECHNOLOGIES TO INVESTIGATE MICROBIAL INTERACTIONS}

Recent advances in high-throughput microfluidics, single-cell genomics/transcriptomics, highthroughput sequencing, as well as high-performance computing now allow the study of single microbial cells and their ecological relationships in greater detail. By combining these technologies and methods, it is possible to start the meticulous work of constructing ecological networks that include validated ecological interactions. In the following sections, we will focus on how single-cell genomics and transcriptomics can be used to prove ecological interactions that have or have not been predicted by association networks. Single-cell genomics and transcriptomics are among the most promising methods for unveiling interactions that require physical contact between taxa. Basically, these techniques rely on sorting single cells using automated or manual methods (a.k.a. micromanipulation), subsequently lysing the cells, extracting DNA or RNA, and amplifying the whole genome (DNA) or transcriptome (RNA) (Fig. 4). Afterwards, the amplified genome or transcriptome can be sequenced using high-throughput sequencing. Obtained genomic or transcriptomic information from microbial holobionts (i.e. a single cell and all its associated symbionts; Box 1) can reveal or prove physical association between different cells. In addition, genomic or transcriptomic data can uncover important genes for symbiotic relationships as well as genes that have co-evolved in both host and symbiont (Zilber-Rosenberg \& Rosenberg 2008, Dheilly 2014).

\section{SINGLE-CELL GENOMICS}

High-throughput single-cell genomics normally involves isolating single cells using fluorescence activated cell sorting (FACS), which allows isolating a large number of cells from, e.g., seawater samples (Stepanauskas 2012) (Fig. 4). After cell lysis, the genomes of individual cells together with their symbionts can be amplified using, e.g., multiple displacement amplification (MDA; Fig. 4) (Lasken 2012, Stepanauskas 2012). The resulting single amplified genomes (SAGs) can then be screened using PCR of specific taxonomic gene-markers coupled to Sanger or Illumina MiSeq sequencing in order to determine physical co-existence of different taxa in the same isolated cell or SAG. For example, sequencing $18 \mathrm{~S}$ (for eukaryotes) or 16S (for prokaryotes) rRNA genes in these SAGs will identify the host as well as the symbionts. The latter approach has been used to explore potential ecological interactions between protist and bacteria in marine communities. MartínezGarcía et al. (2012) sorted single-cells using Lysotracker, which stains food vacuoles in protists, and performed MDA of the DNA originating from the sorted cells plus symbionts. Afterwards, MDA products were used as templates in PCRs that amplified the $18 \mathrm{~S}$ and $16 \mathrm{~S}$ separately. Both $18 \mathrm{~S}$ and $16 \mathrm{~S}$ sequences were obtained for 7 SAGs, which pointed to multiple cases of physical co-existence between the bacterivorous flagellate MAST- 4 and the bacterioplankton SAR11 (arguably the most abundant bacterioplankton in the ocean), between multiple microeukaryotes (Chrysophytes, Apicomplexa, and Basidiomycota) and Bacteroidetes as well as between Paraphysomonas butcheri and Actinobacteria. In the

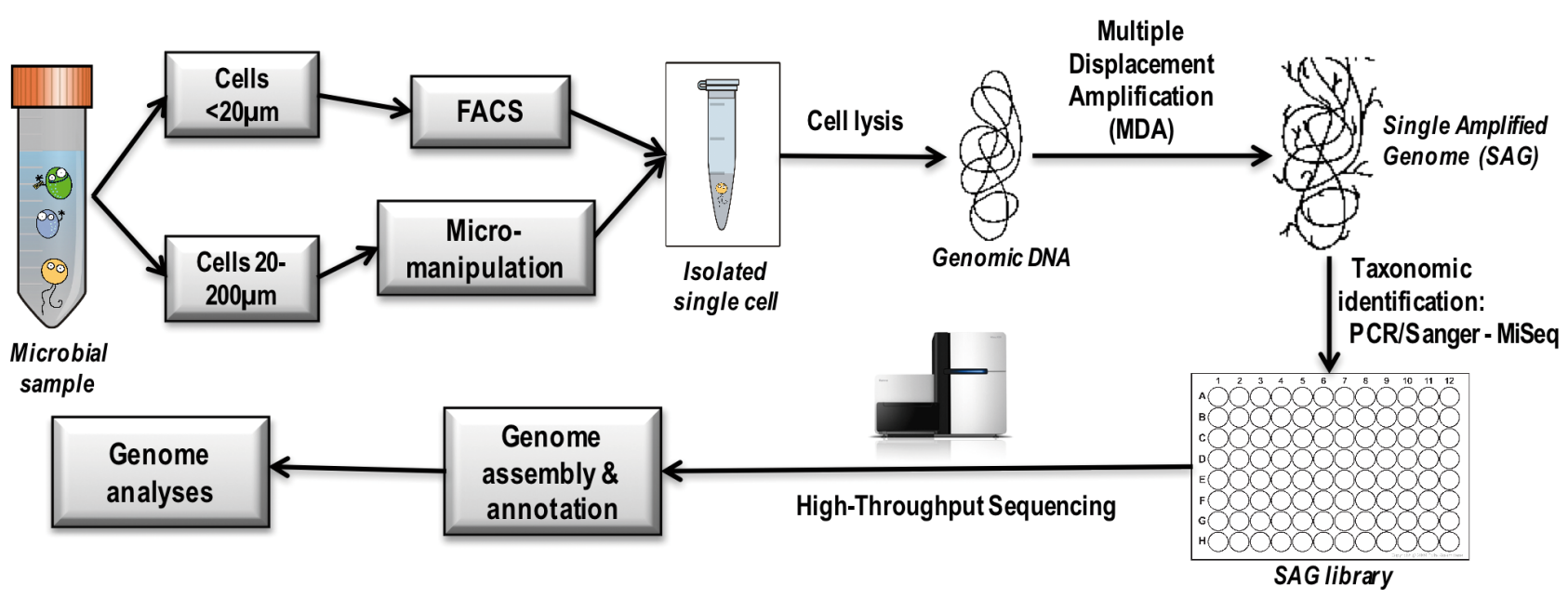

Fig. 4. Description of the single-cell genomics workflow. FACS: fluorescence activated cell sorting 
latter cases, physical co-existence within the same SAG seems to be the outcome of grazing (MartínezGarcía et al. 2012), which should be confirmed by other techniques, such as microscopy.

So far, most studies use direct Sanger sequencing to retrieve $16 \mathrm{~S}$ or $18 \mathrm{~S}$ occurring within the same SAG. This approach will probably not work if multiple $18 \mathrm{~S}$ and/or $16 \mathrm{~S}$ are present within the same SAG (e.g. a protist infected with protist parasites, or a protist harboring multiple bacterial symbionts). To sequence the rDNA in several SAGs that harbor multiple symbionts, multiplexed $18 \mathrm{~S}$ or $16 \mathrm{~S}$ rDNA amplicon sequencing with Illumina MiSeq seems the most suitable option. Another option to validate physical associations is to work with unassembled reads retrieved from different SAGs after shotgun sequencing. 16S or $18 \mathrm{~S}$ rDNA fragments can be detected using hidden Markov models (Logares et al. 2014b) and taxonomically classified, unveiling all taxa present within the same SAG. This approach has been tested by R. Logares (unpubl.) in different MAST-4 SAGs from TARA Oceans, and different bacterial 16S fragments could be detected within different MAST4 SAGs.

Other studies have confronted the challenge of binning contigs from $>1$ genome retrieved from the same SAG. For example, Yoon et al. (2011) analysed assembled sequences from 3 picobiliphyte (protist) SAGs and found viral and bacterial signal, pointing to grazing and viral infection. Nevertheless, the separation of contigs into bins that represent different co-occurring organisms is not straightforward, even though special tools are designed for this, e.g. ProDeGe (Tennessen et al. 2016). New automated methods for binning contigs of different taxa retrieved from the same SAG are urgently needed. These methods are particularly relevant in a context of an increasing interest in sequencing SAGs from organisms that are expected to have symbionts or prey in the process of being digested. Once automated binning is achieved, and assuming a future reduction in sequencing costs, detection of interactions from assembled SAG data may become a routine procedure.

A word of caution might be in order, however, because typical single-cell genomics (Fig. 4) involving bulk MDA has limitations due to uneven amplification of different regions of the genome (Rodrigue et al. 2009). Sequences amplified early in the process tend to be highly overrepresented in the final MDA product. As a consequence, MDA products often have a high coverage of some areas of the genome and low, or no, coverage of other areas (Sidore et al. 2016). This is especially challenging when dealing with eukaryotic organisms because their genomes are larger than bacterial genomes and can have long repetitive stretches. Recent studies have found that genome recovery per SAG for MAST-4 flagellate cells ranged between 20 and 40\% (Roy et al. 2014, Mangot et al. 2017). This means that during traditional MDA-based SAG sequencing, not only is a fraction of the host genome missed, but also the genomes of its symbionts can be underrepresented or totally missed.

The bias in the MDA process can be reduced by performing MDA in emulsion solution or in monodisperse droplets (Sidore et al. 2016). By separating fragments of DNA in nano-litre droplets before amplification, the bias is reduced because the isolated molecules can amplify to saturation without competing for substrates. Other amplification methods, such as MALBAC, DOP-PCR and PicoPLEX (Gawad et al. 2016), are also available, but there is little information so far on their use with protists. In the near future, amplification protocols will likely produce virtually complete and unbiased genomes for many microbial holobionts. Then, it will be possible not only to analyze the different metabolic pathways involved in symbiotic interactions but also to determine to which partner the genes involved belong to.

\section{SINGLE-CELL TRANSCRIPTOMICS}

A complementary approach to genomic analyses of interacting cells is to examine expressed genes through RNA or cDNA sequencing. Research on single-cell transcriptomics has been progressing in biomedicine, driven by the desire to know how cells differ in both their genomes and gene expression, even though they may originate from the same tissue (Crosetto et al. 2015). Cells in a population are not homogenous, and extraction of RNA from several cells or tissues will give an average overview of the expression in the population (Liu et al. 2014, Wu et al. 2014). Yet, extracting and amplifying the transcriptome (mRNA) of a single cell can be more challenging than extracting the DNA. The amount of mRNA that can be isolated from a cell is small, and amplification steps are necessary prior to sequencing. RNA is also less stable than DNA, starting to degrade quite quickly when a cell dies, being destroyed by the still active RNAses in the cell (Jackowiak et al. 2011). Therefore, the lysis protocol has to be adjusted in order to break the cell without destroying the RNA.

In ecology, the interest in single-cell transcriptomics has exploded during the last couple of years. 
Yet, only 1 study on cultured ciliates (Kolisko et al. 2014) and a recent study on natural samples of radiolarian cells (Krabberød et al. 2017) have demonstrated that the approach can generate representative data for the total number of expressed genes. Such transcriptome libraries are goldmines for studies of gene function as well as of genes involved in establishing symbiotic relationships. In a temporal framework, dynamic expression profiles can reflect different stages of the interaction, providing detailed information about the molecular mechanisms involved in establishing and maintaining holobionts (Dheilly 2014). Soon, we should be able to answer questions such as: how can a cell be engulfed but not digested in the case of endosymbiosis, i.e. how can the host perform controlled phagocytosis without breakdown of the symbiont? Furthermore, how does the host control the biomass of the symbiont, but still ensure that it thrives? In the particular case of photosymbionts (Box 1), how is the need for different nutrients communicated between partners?

In sum, single-cell transcriptomics has the potential to provide valuable insight into the genetic mechanisms involved in symbiotic interactions, as for example in the case of coral reefs and dinoflagellates. Many of the genetic mechanisms in this specific interaction are known (Schwarz et al. 2008, Davy et al. 2012, Meyer \& Weis 2012), but contributions of individual cells are unknown. Thus, single-cell transcriptomics could help determine what different cells contribute in this interaction. Single-cell transcriptomics can also uncover the genetic machinery involved in the different stages of parasitic infection. For instance, it would be possible to isolate single dinoflagellate cells infected with Amoebophrya (Coats \& Park 2002, Chambouvet et al. 2008) in each of the different stages of the infection to determine how the parasite attacks the host and how the host responds to the attack. Furthermore, single-cell transcriptomics could be used to analyze both eukaryotic and prokaryotic transcripts occurring in a microholobiont (Kang et al. 2011), thus allowing us to explore cross-domain interactions at the intra-cellular scale.

\section{CONCLUSION}

Understanding microbial interactions in the oceans will prove a major challenge in microbial ecology over the next decade. Luckily, there are promising developments in both sequencing technologies and in molecular methods for working with genomes and transcriptomes at the single-cell level, which will continue to develop and improve. In particular, the field of single-cell metaomics is advancing quickly, and in a short time, it will likely be possible to routinely sequence both the transcriptome and the genome of a single cell. One such method has already been tested on samples from a mouse embryo (Dey et al. 2015), and it should in theory be possible to adapt this method to protists. In sum, coupling big data originating from metaomics approaches together with new bioinformatic algorithms and high-performance computing will contribute to a much better understanding of the oceanic microeukaryotic interactome and its relationships with the functioning of the biosphere.

Acknowledgements. This work has been supported by the Research Council of Norway (project 240904). We thank Clara Ruiz González for the drawing of microbes as well as Ina Deutschmann, the 2 reviewers and the AME editor for useful comments and suggestions that helped to improve this work.

\section{LITERATURE CITED}

Amend AS, Barshis DJ, Oliver TA (2012) Coral-associated marine fungi form novel lineages and heterogeneous assemblages. ISME J 6:1291-1301

Amundsen PA, Lafferty KD, Knudsen R, Primicerio R, Klemetsen A, Kuris AM (2009) Food web topology and parasites in the pelagic zone of a subarctic lake. J Anim Ecol 78:563-572

Anderson OR (1983) Radiolaria. Springer-Verlag, New York, NY

Barabasi AL, Albert R (1999) Emergence of scaling in random networks. Science 286:509-512

*Bascompte J (2009) Disentangling the web of life. Science 325:416-419

* Berney C, Romac S, Mahe F, Santini S, Siano R, Bass D (2013) Vampires in the oceans: predatory cercozoan amoebae in marine habitats. ISME J 7:2387-2399

* Bråte J, Krabberød AK, Dolven JK, Ose RF, Kristensen T, Bjørklund KR, Shalchian-Tabrizi K (2012) Radiolaria associated with large diversity of marine alveolates. Protist 163:767-777

Breitbart M (2012) Marine viruses: truth or dare. Annu Rev Mar Sci 4:425-448

Brum JR, Ignacio-Espinoza JC, Roux S, Doulcier G and others (2015) Patterns and ecological drivers of ocean viral communities. Science 348:1261498

Chambouvet A, Morin P, Marie D, Guillou L (2008) Control of toxic marine dinoflagellate blooms by serial parasitic killers. Science 322:1254-1257

Chow CE, Kim DY, Sachdeva R, Caron DA, Fuhrman JA (2014) Top-down controls on bacterial community structure: microbial network analysis of bacteria, T4-like viruses and protists. ISME J 8:816-829

Coats D, Park M (2002) Parasitism of photosynthetic dinoflagellates by three strains of Amoebophrya (Dinophyta): parasite survival, infectivity, generation time, and host specificity. J Phycol 38:520-528 
Crosetto N, Bienko M, Oudenaarden AV (2015) Spatially resolved transcriptomics and beyond. Nat Rev Genet 16: 57-66

Darwin C (1859) On the origin of species by means of natural selection. J. Murray, London

Davy SK, Allemand D, Weis VM (2012) Cell biology of cnidarian-dinoflagellate symbiosis. Microbiol Mol Biol Rev 76:229-261

de Bary A (1879) Die Erscheinung der Symbiose. Verlag von Karl J. Trubner, Strassburg

de Meeûs T, Renaud F (2002) Parasites within the new phylogeny of eukaryotes. Trends Parasitol 18:247-251

de Vargas C, Audic S, Henry N, Decelle J and others (2015) Eukaryotic plankton diversity in the sunlit ocean. Science 348:1261605

Decelle J, Romac S, Sasaki E, Not F, Mahé F (2014) Intracellular diversity of the V4 and V9 regions of the $18 \mathrm{~S}$ rRNA in marine protists (radiolarians) assessed by highthroughput sequencing. PLOS One 9(8):e104297

del Campo J, Pombert JF, Slapeta J, Larkum A, Keeling PJ (2016) The 'other' coral symbiont: Ostreobium diversity and distribution. ISME J 11:296-299

DeLong EF, Karl DM (2005) Genomic perspectives in microbial oceanography. Nature 437:336-342

* Dey SS, Kester L, Spanjaard B, Bienko M, van Oudenaarden A (2015) Integrated genome and transcriptome sequencing of the same cell. Nat Biotechnol 33:285-289

* Dheilly NM (2014) Holobiont-holobiont interactions: redefining host-parasite interactions. PLoS Pathog 10: e1004093

Wolven JK, Lindqvist C, Albert VA, Bjorklund KR, Yuasa T, Takahashi O, Mayama S (2007) Molecular diversity of alveolates associated with neritic North Atlantic radiolarians. Protist 158:65-76

* Dunne JA, Lafferty KD, Dobson AP, Hechinger RF and others (2013) Parasites affect food web structure primarily through increased diversity and complexity. PLoS Biol 11:e1001579

Edgar RC (2013) UPARSE: highly accurate OTU sequences from microbial amplicon reads. Nat Methods 10: 996-998

Faust K, Raes J (2012) Microbial interactions: from networks to models. Nat Rev Microbiol 10:538-550

Fredrickson AG, Stephanopoulos G (1981) Microbial competition. Science 213:972-979

* Gast RJ, Caron DA (1996) Molecular phylogeny of symbiotic dinoflagellates from planktonic foraminifera and radiolaria. Mol Biol Evol 13:1192-1197

Gause GF (1934) Experimental analysis of Vito Volterra's mathematical theory of the struggle for existence. Science 79:16-17

Gawad C, Koh W, Quake SR (2016) Single-cell genome sequencing: current state of the science. Nat Rev Genet 17: 175-188

* Gómez F, Simão TLL, Utz LRP, Lopes RM (2016) The nature of the diatom Leptocylindrus mediterraneus (Bacillariophyceae), host of the enigmatic symbiosis with the stramenopile Solenicola setigera. Phycologia 55:265-273

Goodwin S, McPherson JD, McCombie WR (2016) Coming of age: ten years of next-generation sequencing technologies. Nat Rev Genet 17:333-351

Guerrero R, Pedros-Alio C, Esteve I, Mas J, Chase D, Margulis L (1986) Predatory prokaryotes: predation and primary consumption evolved in bacteria. Proc Natl Acad Sci USA 83:2138-2142
Guillou L, Viprey M, Chambouvet A, Welsh RM and others (2008) Widespread occurrence and genetic diversity of marine parasitoids belonging to Syndiniales (Alveolata). Environ Microbiol 10:3349-3365

*Harada A, Ohtsuka S, Horiguchi T (2007) Species of the parasitic genus Duboscquella are members of the enigmatic Marine Alveolate Group I. Protist 158:337-347

* Ikenoue T, Bjørklund KR, Dumitrica P, Krabberød AK, Kimoto K, Matsuno K, Harada N (2016) Two new living Entactinaria (Radiolaria) species from the Arctic province: Joergensenium arcticum n. sp. and Joergensenium clevei n. sp. Mar Micropaleontol 124:75-94

Jackowiak P, Nowacka M, Strozycki PM, Figlerowicz M (2011) RNA degradome-its biogenesis and functions. Nucleic Acids Res 39:7361-7370

Jephcott TG, Sime-Ngando T, Gleason FH, Macarthur DJ (2016) Host-parasite interactions in food webs: diversity, stability, and coevolution. Food Webs 6:1-8

Jolley ET, Jones AK (1977) The interaction between Navicula muralis Grunow and an associated species of Flavobacterium. Br Phycol J 12:315-328

* Kang Y, Norris MH, Zarzycki-Siek J, Nierman WC, Donachie SP, Hoang TT (2011) Transcript amplification from single bacterium for transcriptome analysis. Genome Res 21:925-935

Karsenti E, Acinas SG, Bork P, Bowler C and others (2011) A holistic approach to marine eco-systems biology. PLOS biol 9:e1001177

Kirchman DL (ed) (2008) Microbial ecology of the oceans, 2nd edn. John Wiley \& Sons, Hoboken, NJ

Knoll AH (2014) Paleobiological perspectives on early eukaryotic evolution. Cold Spring Harb Perspect Biol 6: a016121

Kolisko M, Boscaro V, Burki F, Lynn DH, Keeling PJ (2014) Single-cell transcriptomics for microbial eukaryotes. Curr Biol 24:R1081-R1082

Krabberød AK, Orr RJS, Bråte J, Kristensen T, Bjorklund KR, Shalchian-Tabrizi K (2017) Single cell transcriptomics, mega-phylogeny and the genetic basis of morphological innovations in Rhizarid. Mol Biol Evol msx075

*Lasken RS (2012) Genomic sequencing of uncultured microorganisms from single cells. Nat Rev Microbiol 10:631-640

Leadbetter ER, Poindexter JS (1986) Bacteria in nature: methods and special applications in bacterial ecology, Vol 2. Springer-Verlag, New York, NY

* Legrand C, Rengefors K, Fistarol GO, Graneli E (2003) Allelopathy in phytoplankton - biochemical, ecological and evolutionary aspects. Phycologia 42:406-419

Lidicker WZA (1979) Clarification of interactions in ecological systems. Bioscience 29:475-477

Kima-Mendez G, Faust K, Henry N, Decelle J and others (2015) Ocean plankton. Determinants of community structure in the global plankton interactome. Science 348:1262073

Liu N, Liu L, Pan X (2014) Single-cell analysis of the transcriptome and its application in the characterization of stem cells and early embryos. Cell Mol Life Sci 71: 2707-2715

Locey KJ, Lennon JT (2016) Scaling laws predict global microbial diversity. Proc Natl Acad Sci USA 113: 5970-5975

Logares R (2006) Does the global microbiota consist of a few cosmopolitan species? Ecol Austral 16:85-90

* Logares R, Haverkamp TH, Kumar S, Lanzen A, Nederbragt AJ, Quince C, Kauserud H (2012) Environmental micro- 
biology through the lens of high-throughput DNA sequencing: synopsis of current platforms and bioinformatics approaches. J Microbiol Methods 91:106-113

* Logares R, Lindstrom ES, Langenheder S, Logue JB and others (2013) Biogeography of bacterial communities exposed to progressive long-term environmental change. ISME J 7:937-948

Logares R, Audic S, Bass D, Bittner L and others (2014a) Patterns of rare and abundant marine microbial eukaryotes. Curr Biol 24:813-821

Logares R, Sunagawa S, Salazar G, Cornejo-Castillo FM and others (2014b) Metagenomic 16S rDNA Illumina tags are a powerful alternative to amplicon sequencing to explore diversity and structure of microbial communities. Environ Microbiol 16:2659-2671

Logares R, Mangot JF, Massana R (2015) Rarity in aquatic microbes: placing protists on the map. Res Microbiol 166: 831-841

* López-García P, Moreira D (2008) Tracking microbial biodiversity through molecular and genomic ecology. Res Microbiol 159:67-73

MacArthur RH, Wilson EO (1967) The theory of island biogeography. Princeton University Press, Princeton, NJ

* Mangot JF, Logares R, Sanchez P, Latorre F and others (2017) Accessing the genomic information of unculturable oceanic picoeukaryotes by combining multiple single cells. Sci Rep 7:41498

Margulis L, Fester R (1991) Symbiosis as a source of evolutionary innovation: speciation and morphogenesis, Vol 11. MIT Press, Cambridge, MA

Martin BD, Schwab E (2013) Current usage of symbiosis and associated terminology. Int J Biol 5:32-45

* Martínez-García M, Brazel D, Poulton NJ, Swan BK and others (2012) Unveiling in situ interactions between marine protists and bacteria through single cell sequencing. ISME J 6:703-707

*Martínez-García M, Santos F, Moreno-Paz M, Parro V, Anton J (2014) Unveiling viral-host interactions within the 'microbial dark matter'. Nat Commun 5:4542

* Massana R (2011) Eukaryotic picoplankton in surface oceans. Annu Rev Microbiol 65:91-110

Massana R, Gobet A, Audic S, Bass D and others (2015) Marine protist diversity in European coastal waters and sediments as revealed by high-throughput sequencing. Environ Microbiol 17:4035-4049

Meyer E, Weis VM (2012) Study of cnidarian-algal symbiosis in the 'omics' age. Biol Bull 223:44-65

* Miller TR, Belas R (2004) Dimethylsulfoniopropionate metabolism by Pfiesteria-associated Roseobacter spp. Appl Environ Microbiol 70:3383-3391

Montoya JM, Pimm SL, Solé RV (2006) Ecological networks and their fragility. Nature 442:259-264

*Moon-van der Staay SY, De Wachter R, Vaulot D (2001) Oceanic 18S rDNA sequences from picoplankton reveal unsuspected eukaryotic diversity. Nature 409:607-610

Mordret S, Romac S, Henry N, Colin S and others (2016) The symbiotic life of Symbiodinium in the open ocean within a new species of calcifying ciliate (Tiarina sp.). ISME J 10:1424-1436

* Not F, Gausling R, Azam F, Heidelberg JF, Worden AZ (2007) Vertical distribution of picoeukaryotic diversity in the Sargasso Sea. Environ Microbiol 9:1233-1252

Not F, del Campo J, Balague V, de Vargas C, Massana R (2009) New insights into the diversity of marine picoeukaryotes. PLOS ONE 4:e7143
Nowack EC, Melkonian M (2010) Endosymbiotic associations within protists. Philos Trans R Soc Lond B 365: $699-712$

*Parfrey LW, Lahr DJG, Knoll AH, Katz LA (2011) Estimating the timing of early eukaryotic diversification with multigene molecular clocks. Proc Natl Acad Sci USA 108: 13624-13629

Pascual M, Dunne JA (2006) Ecological networks. Oxford University Press, New York, NY

*Patterson DJ (1999) The diversity of eukaryotes. Am Nat 154:S96-S124

*Pawlowski J, Holzmann M, Fahrni JF, Pochon X, Lee JJ (2001) Molecular identification of algal endosymbionts in large miliolid Foraminifera: 2. Dinoflagellates. J Eukaryot Microbiol 48:368-373

*Pedrós-Alió C (2012) The rare bacterial biosphere. Annu Rev Mar Sci 4:449-466

*Pernice MC, Logares R, Guillou L, Massana R (2013) General patterns of diversity in major marine microeukaryote lineages. PLOS ONE 8:e57170

* Porter SM (2016) Tiny vampires in ancient seas: evidence for predation via perforation in fossils from the 780-740 million-year-old Chuar Group, Grand Canyon, USA. Proc R Soc B 283:20160221

* Poulin R, Randhawa HS (2015) Evolution of parasitism along convergent lines: from ecology to genomics. Parasitology 142:S6-S15

Raes J, Bork P (2008) Molecular eco-systems biology: towards an understanding of community function. Nat Rev Microbiol 6:693-699

* Rinke C, Schwientek P, Sczyrba A, Ivanova NN and others (2013) Insights into the phylogeny and coding potential of microbial dark matter. Nature 499:431-437

* Rodrigue S, Malmstrom RR, Berlin AM, Birren BW, Henn MR, Chisholm SW (2009) Whole genome amplification and de novo assembly of single bacterial cells. PLOS ONE 4:e6864

Rohwer F, Thurber RV (2009) Viruses manipulate the marine environment. Nature 459:207-212

* Roy RS, Price DC, Schliep A, Cai G and others (2014) Single cell genome analysis of an uncultured heterotrophic stramenopile. Sci Rep 4:4780

* Sañudo-Wilhelmy SA, Gomez-Consarnau L, Suffridge C, Webb EA (2014) The role of B vitamins in marine biogeochemistry. Annu Rev Mar Sci 6:339-367

* Schloss PD, Girard RA, Martin T, Edwards J, Thrash JC (2016) Status of the archaeal and bacterial census: an update. MBio 7:e00201-e00216

* Schwarz JA, Brokstein PB, Voolstra C, Terry AY and others (2008) Coral life history and symbiosis: functional genomic resources for two reef building Caribbean corals, Acropora palmata and Montastraea faveolata. BMC Genomics 9:97

Sherr EB, Sherr BF (2002) Significance of predation by protists in aquatic microbial food webs. Ant Leeuwenhoek 81:293-308

Siano R, Montresor M, Probert I, Not F, de Vargas C (2010) Pelagodinium gen. nov. and $P$. beii comb. nov., a dinoflagellate symbiont of planktonic foraminifera. Protist 161: 385-399

Sidore AM, Lan F, Lim SW, Abate AR (2016) Enhanced sequencing coverage with digital droplet multiple displacement amplification. Nucleic Acids Res 44:e66

Sinclair L, Osman OA, Bertilsson S, Eiler A (2015) Microbial community composition and diversity via $16 \mathrm{~S}$ rRNA gene 
amplicons: evaluating the Illumina platform. PLOS ONE 10:e0116955

Skovgaard A (2014) Dirty tricks in the plankton: diversity and role of marine parasitic protists. Acta Protozool 53:51-62

Skovgaard A, Daugbjerg N (2008) Identity and systematic position of Paradinium poucheti and other Paradiniumlike parasites of marine copepods based on morphology and nuclear-encoded SSU rDNA. Protist 159:401-413

Skovgaard A, Meneses I, Angélico MM (2009) Identifying the lethal fish egg parasite Ichthyodinium chabelardi as a member of Marine Alveolate Group I. Environ Microbiol 11:2030-2041

Sogin ML, Morrison HG, Huber JA, Mark Welch D and others (2006) Microbial diversity in the deep sea and the underexplored 'rare biosphere'. Proc Natl Acad Sci USA 103:12115-12120

Stat M, Morris E, Gates RD (2008) Functional diversity in coral-dinoflagellate symbiosis. Proc Natl Acad Sci USA 105:9256-9261

Steele JA, Countway PD, Xia L, Vigil PD and others (2011) Marine bacterial, archaeal and protistan association networks reveal ecological linkages. ISME J 5:1414-1425

Stepanauskas R (2012) Single cell genomics: an individual look at microbes. Curr Opin Microbiol 15:613-620

Suttle CA (2007) Marine viruses-major players in the global ecosystem. Nat Rev Microbiol 5:801-812

Editorial responsibility: Eva Lindström,

Uppsala, Sweden
Tennessen K, Andersen E, Clingenpeel S, Rinke C and others (2016) ProDeGe: a computational protocol for fully automated decontamination of genomes. ISME J 10: 269-272

*Wagner-Döbler I, Ballhausen B, Berger M, Brinkhoff T and others (2010) The complete genome sequence of the algal symbiont Dinoroseobacter shibae: a hitchhiker's guide to life in the sea. ISME J 4:61-77

*Watts DJ, Strogatz SH (1998) Collective dynamics of 'smallworld' networks. Nature 393:440-442

*Wilkinson DM (2001) At cross purposes. Nature 412:485

* Wu AR, Neff NF, Kalisky T, Dalerba P and others (2014) Quantitative assessment of single-cell RNA-sequencing methods. Nat Methods 11:41-46

* Yoon HS, Price DC, Stepanauskas R, Rajah VD and others (2011) Single-cell genomics reveals organismal interactions in uncultivated marine protists. Science 332: 714-717

Khu F, Massana R, Not F, Marie D, Vaulot D (2005) Mapping of picoeucaryotes in marine ecosystems with quantitative PCR of the 18S rRNA gene. FEMS Microbiol Ecol 52: 79-92

Zilber-Rosenberg I, Rosenberg E (2008) Role of microorganisms in the evolution of animals and plants: the hologenome theory of evolution. FEMS Microbiol Rev 32: 723-735

Submitted: July 22, 2016; Accepted: December 7, 2016

Proofs received from author(s): March 19, 2017 